\section{Ahmed G, Mohammed SS, Abdulkadir MB, Adesiyun $\mathrm{OO}$}

\title{
Ventilatory support of the newborn
}

DOI:http://dx.doi.org/10.4314/njp.v39i1.8

\begin{tabular}{l} 
Received: 3rd November 2011 \\
Accepted: 3rd November 2011 \\
\hline
\end{tabular}

Adesiyun OO $(\square)$

Ahmed G,

Mohammed SS,

Abdulkadir MB,

Department of Paediatrics and Child Health, University of Ilorin Teaching Hospital,

P.M.B.1459, Ilorin, Nigeria.

Email: omotayoadesiyun@yahoo.com, Tel: +2347087621125
Abstract Respiratory disorders are a frequent cause of admission in the newborn. Respiratory diseases have unique physiologic, anatomic and clinical characteristics during this period necessitating special management. $\mathrm{K} \mathrm{n}$ o w l e d g e of $\mathrm{th}$ e pathophysiology of pulmonary diseases and their differential impact on the lungs of differing stages of maturity is essential to the safe and efficacious applications of special techniques of treatment. Principles of respiratory $\mathrm{mana} g$ e ment i n clude establishment of the airway, ensuring oxygenation, assisted ventilation, assessing adequacy of ventilation, correction of metabolic abnormalities and alleviation of cause of distress.
The goal of treatment is safe and effective assistance to oxygen delivery and carbon dioxide removal from the tissues. Inspired oxygen should be administered in a controlled manner to provide adequate but not excessive blood oxygen tension levels. Mechanical ventilation may be required to treat metabolic abnormalities. There is the need for continuous monitoring and re- evaluation. This article is intended to present an overview of the embryology of the respiratory system, pulmonary physiology in the newborn, the principles of oxygen therapy and mechanical ventilation. It also discusses the complications that can follow.

Key words: Ventilatory support, oxygen delivery, newborn

\section{Introduction}

\section{Embryology of the Respiratory System}

The respiratory system is an outgrowth of the ventral wall of the foregut. While the epithelium of the larynx, tracheal, bronchi, and the alveoli originate in the endoderm, the cartilaginous, muscular, and connective tissue component are mesodermal in origin. By the $4^{\text {th }}$ week of gestation, the tracheoesophageal septum separates the tracheal from the foregut, with the foregut gut dividing as the lung bud anteriorly and the esophagus posteriorly. Contact between the two is maintain through the larynx, which is formed by the tissues of the $4^{\text {th }}$ and $6^{\text {th }}$ pharyngeal arches. The lung bud develop into 2 main bronchi, while the right forms 3 secondary bronchi and 3 lobes, the left forms 2 bronchi and 2 lobes.
The development of the lung is divided into 5 overlapping stages.

Embryonic stage (3-7week) branching of the primitive bud to form terminal bronchioles.

Pseudo glandular stage (5-17weeks) further division of the terminal bronchiole into 2 or more respiratory bronchioles.

Canalicular stage (16-26week) terminal sacs are form and the capillaries establishes close network. The type II alveoli cells are well delineated.

Saccular stage (26-36week) thinning of the interstitium and fusion of type I cells, and the capillary basement in preparation for the lung function as an organ of gas exchange.

Alveolar stage (36week -3-8yrs of age) secondary septal formation, further sprouting of the capillary network \& development of true alveoli. 
Towards the end of the $6^{\text {th }}$ month, type II alveoli cells produce surfactant a phospholipids rich fluid capable of lowering surface tension at the alveoli surface.

\section{Clinical importance}

Abnormalities in stage I lung aplasia, tracheoesophageal fistula and pulmonary cysts.

Abnormalities in stage II - pulmonary sequestration, cystic adenomatoid malformations, congenital diaphragmatic hernia.

Abnormalities in stage III - Respiratory distress syndrome (RDS) and lung hypoplasia.

Before birth, the lungs are filled with fluid that has a high chloride content, little protein, some mucus from bronchi gland. Fetal breathing movement begins before birth and these movements are important for stimulating the development and conditioning of respiratory muscles.

\section{Pulmonary Physiology in the Newborn}

The goals of respiration are to provide oxygen to the tissues and to remove carbon dioxide. To achieve these goals, respiration occurs through four major functions:

Pulmonary ventilation which involves the inflow and outflow of air between the atmosphere and the lung alveoli;

Diffusion of oxygen and carbon dioxide between the alveoli and the blood gas exchange.

Transport of oxygen and carbon dioxide in the blood and body fluids to and from the body's tissue cells.

Regulation of ventilation and other facets of respiration.

\section{Mechanics of Pulmonary Ventilation}

The lungs can be expanded and contracted in two ways: Either by downward and upward movement of the diaphragm to lengthen or shorten the chest cavity or via elevation and depression of the ribs to increase and decrease the anteroposterior diameter of the chest cavity. Normal quiet breathing is accomplished almost entirely by the first method while the second method occurs during heavy breathing and involves the use of muscles of inspiration and expiration.

Pleural pressure is the pressure of the fluid in the thin space between the lung pleura and the chest wall pleura. This is normally a slight negative pressure. On the other hand, alveolar pressure is the pressure of the air inside the lung alveoli. When no air is flowing into or out of the lungs, the pressures in all parts of the respiratory tree, all the way to the alveoli, are equal to atmospheric pressure, that is, $0 \mathrm{cmH}_{2} \mathrm{O}$ pressure. Transpulmonary pressure is the difference between the alveolar pressure and the pleural pressure. It is the pressure difference between that in the alveoli and that on the outer surfaces of the lungs, and it is a measure of the elastic forces in the lungs that tend to collapse the lungs at each instant of respiration, called the recoil pressure.

Compliance describes the elasticity or distensibility (e.g., of the lungs, chest wall, respiratory system) and is calculated from the change in volume per unit change in pressure as follows:

Compliance $=\Delta$ Volume

\section{$\Delta$ Pressure}

The higher the compliance, the larger the delivered volume per unit changes in pressure. Normally, the chest wall is compliant in newborns and does not impose a substantial elastic load compared to the lungs. The range of total respiratory system compliance (lungs + chest wall) in newborns with healthy lungs is $0.003-0.006 \mathrm{~L} / \mathrm{cm} \mathrm{H}_{2} \mathrm{O}$, while compliance in babies with RDS may be as low as $0.0005-0.001 \mathrm{~L} / \mathrm{cm} \mathrm{H}_{2} \mathrm{O}$. The alveolar surface tension is an important factor affecting the compliance of the lungs is the surface tension of the film of fluid that lines the alveoli. If the surface tension is not kept low when the alveoli become smaller during expiration, they collapse in accordance with the law of Laplace.

The law states that in spherical structures like the alveoli, the distending pressure equals 2 times the tension divided by the radius $(\mathrm{P}=2 \mathrm{~T} / \mathrm{r})$. The low surface tension when the alveoli are small is due to the presence in the fluid lining the alveoli of surfactant, a lipid surface-tension-lowering agent. Surfactant is a mixture of dipalmitoylphosphatidyl-choline (DPPC), other lipids, and proteins. Surfactant also helps to prevent pulmonary edema.

Resistance describes the inherent capacity of the air conducting system (e.g, airways, endotracheal tube [ETT]) and tissues to oppose airflow and is expressed as the change in pressure per unit change in flow as follows:

Resistance $=\triangleq$ Pressure

\section{$\triangle$ Flow}

Airway resistance depends on the radii of the airways (total cross-sectional area), the length of airways, the flow rate, and the density and viscosity of gas. Resistance is governed by Poiseuille's law stated as:

$\mathrm{R}=81 \eta \div \pi \mathrm{r}^{4}$ (where $\mathrm{R}$ - resistance, $\eta$ - viscosity, 1 - length, and $\mathrm{r}$ - radius). 
Thus, airway resistance is inversely proportional to its radius raised to the $4^{\text {th }}$ power. If the airway lumen is decreased in half, the resistance will increase 16fold. Newborns and young infants with their inherently smaller airways are especially prone to marked increase in airway resistance from inflamed tissues and secretions. In diseases in which airway resistance is increased, flow often becomes turbulent. Distal airways normally contribute less to airway resistance because of their larger crosssectional area, unless bronchospasm, mucosal edema, and interstitial edema decrease their lumen. Small endotracheal tubes that may contribute significantly to airway resistance also are important, especially when high flow rates that lead to turbulent flow are used. The range for total airway plus tissue respiratory resistance values for healthy newborns is $20-40 \mathrm{~cm} \mathrm{H}_{2} \mathrm{O} / \mathrm{L} / \mathrm{s}$; in intubated newborns this range is $50-150 \mathrm{~cm} \mathrm{H}_{2} \mathrm{O} / \mathrm{L} / \mathrm{s}$.

Time constant, measured in seconds, is a product of compliance and resistance.

\section{Time constant $=$ Resistance $X$ Compliance}

It is a measure of how quickly the lungs can inflate or deflate, and also a measure of how quickly the alveoli get into equilibrium with the air passages. Thus, the time constant of the respiratory system is proportional to the compliance and the resistance. For example, the lungs of a healthy newborn with a compliance of $0.004 \mathrm{~L} / \mathrm{cm} \mathrm{H}_{2} \mathrm{O}$ and a resistance of 30 $\mathrm{cm} \mathrm{H}_{2} \mathrm{O} / \mathrm{L} / \mathrm{s}$ have a time constant of 0.12 seconds. When a longer time is allowed for equilibration, a higher percentage of airway pressure equilibrates throughout the lungs. The longer the duration of the inspiratory (or expiratory) time allowed for equilibration, the higher the percentage of equilibration.

\section{For practical purposes:}

One time constant $=63 \%$ equilibrium.

2 time constant $=86 \%$ equilibrium.

3 time constant $=95 \%$ equilibrium.

5 time constant $=100 \%$ equilibrium.

Lungs with decreased compliance (such as in RDS) have a shorter time constant. Lungs with a shorter time constant will complete inflation and deflation faster than normal lungs. Patients with shorter time constants are best ventilated with relatively smaller tidal volumes and faster rates to minimize peak inflation pressure. In patients with increased airway resistance, a fast respiratory rate (and, therefore, less time) does not allow enough pressure equilibration to occur between the proximal airway and the alveoli.

\section{Volumes and Capacities}

Tidal volume $\left(\mathrm{V}_{\mathrm{T}}\right)$ is the amount of air moved in and out of the lungs during each breath. At rest, it is usually $67 \mathrm{~mL} / \mathrm{kg}$ body weight.

Inspiratory capacity (IC) is the amount of air inspired by maximum inspiratory effort after tidal expiration.

Expiratory reserve volume (ERV) is the amount of air exhaled by maximum expiratory effort after tidal expiration.

Residual volume (RV) is the volume of gas remaining in the lungs after maximum expiration.

Vital capacity (VC) is defined as the amount of air moved in and out of the lungs with maximum inspiration and expiration.

Total lung capacity (TLC) is the volume of gas occupying the lungs after maximum inhalation.

Functional residual capacity (FRC) is the amount of air left in the lungs after tidal expiration.

The VC, IC, and ERV are decreased in lung pathology but are also effort dependent. The FRC represents the environment available for pulmonary capillary blood for gas exchange at all times. A decrease in FRC is often encountered in alveolar interstitial diseases and thoracic deformities. The major pathophysiologic consequence of decreased FRC is hypoxemia. Reduced FRC results in a sharp decline in $\mathrm{P}_{\mathrm{A}} \mathrm{O}_{2}$ during exhalation because a limited volume is available for gas exchange.

Gaseous exchange in the respiratory system occurs only in the terminal portions of the airways. The gas that occupies the rest of the respiratory system that is not available for gas exchange with pulmonary capillary blood dead space. This space can be divided as the anatomic dead space (respiratory system volume exclusive of alveoli) and the physiologic (total) dead space (volume of gas not equilibrating with blood, i.e, wasted ventilation). In healthy individuals, the two dead spaces are identical; but in disease states, there may be no exchange between the gas in some of the alveoli and the blood, and some of the alveoli may be overventilated.

Ventilation perfusion (V/Q) mismatch usually is caused by poor ventilation of alveoli relative to their perfusion. A V/Q mismatch is a major cause of hypoxemia in infants with respiratory distress syndrome (RDS) and other causes of respiratory failure.

\section{Gas exchange}

The minute volume is a product of $\mathrm{V}_{\mathrm{T}}$ and respiratory rate. Alveolar ventilation is the volume of atmospheric air entering the alveoli and is calculated as: 
$\left(\mathrm{V}_{\mathrm{T}}\right.$ - dead space $) \times$ respiratory rate

Gas exchange occurs by the process of diffusion and equilibration of alveolar gas with pulmonary capillary blood.

Diffusion depends on the alveolar capillary barrier and amount of available time for equilibration. In health, the equilibration of alveolar gas and pulmonary capillary blood is complete for both oxygen and carbon dioxide. In diseases in which alveolar capillary barrier is abnormally increased (alveolar interstitial diseases) and/or when the time available for equilibration is decreased (increased blood flow velocity), diffusion is incomplete. Because of its greater solubility in liquid medium, carbon dioxide is 20 times more diffusible than oxygen. Significant elevation of $\mathrm{CO}_{2}$ does not occur as a result of a diffusion defect unless there is coexistent hypoventilation.

\section{Oxygen transport}

Oxygen $\left(\mathrm{O}_{2}\right)$ diffuses through the respiratory membrane from the alveoli to the blood from where it is transported to the tissues for utilization. $\mathrm{O}_{2}$ is transported in the blood combined with the $\mathrm{O}_{2}$ carrying protein- hemoglobin. $\mathrm{O}_{2}$ delivery to a particular tissue depends on the amount of $\mathrm{O}_{2}$ entering the lungs, the adequacy of pulmonary gas exchange, the blood flow to the tissue, and the capacity of the blood to carry $\mathrm{O}_{2}$. The reaction is rapid, requiring less than $0.01 \mathrm{~s}$. The deoxygenation (reduction) of $\mathrm{Hb}_{4} \mathrm{O}_{8}$ is also very rapid. The transition from one state to another (i.e deoxyHb $\rightarrow$ $\mathrm{OxyHb} \rightarrow$ deoxyHb) has been calculated to occur about $10^{8}$ times in the life of a red blood cell.

The oxygen-hemoglobin dissociation curve, the curve relating percentage saturation of the $\mathrm{O}_{2}$ carrying power of hemoglobin to the $\mathrm{PO}_{2}$ has a characteristic sigmoid shape. Combination of the first heme in the $\mathrm{Hb}$ molecule with $\mathrm{O}_{2}$ increases the affinity of the second heme for $\mathrm{O}_{2}$, and oxygenation of the second increases the affinity of the third, etc, so that the affinity of hemoglobin for the fourth $\mathrm{O}_{2}$ molecule is many times that for the first.

Three important conditions affect the oxygenhemoglobin dissociation curve: the $\mathrm{pH}$, the temperature, and the concentration of 2, 3diphosphoglycerate (DPG; 2, 3-DPG). A rise in temperature or a fall in $\mathrm{pH}$ shifts the curve to the right. When the curve is shifted in this direction, a higher $\mathrm{PO}_{2}$ is required for hemoglobin to bind a given amount of $\mathrm{O}_{2}$. A convenient index of such shifts is the $\mathrm{P}_{50}$, the $\mathrm{PO}_{2}$ at which hemoglobin is half saturated with $\mathrm{O}_{2}$. Thus, the higher the $\mathrm{P}_{50}$, the lower the affinity of hemoglobin for $\mathrm{O}_{2}$. The greater affinity of fetal hemoglobin (hemoglobin F) than adult hemoglobin (hemoglobin A) for $\mathrm{O}_{2}$ facilitates the movement of $\mathrm{O}_{2}$ from the mother to the fetus.

\section{$\mathrm{O}_{2} \mathrm{Hb}$ dissociation curve}

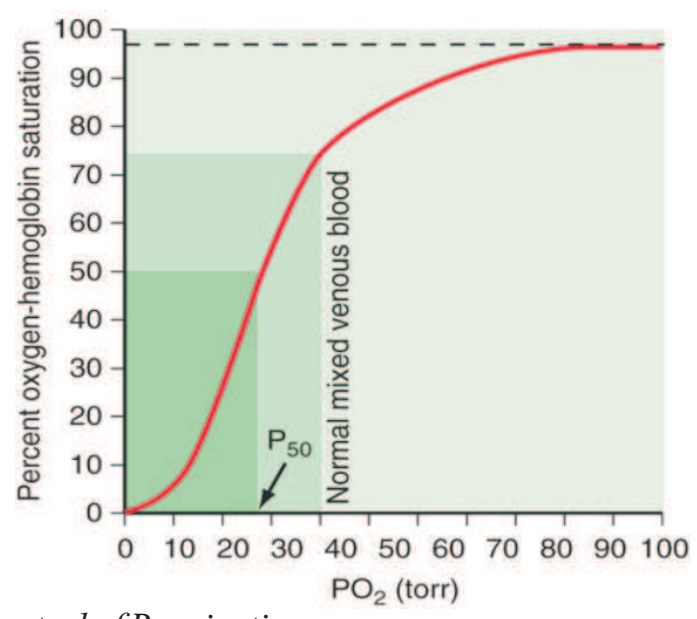

Control of Respiration

The control and maintenance of normal breathing largely reside within the respiratory control centers of the bulbopontine region of the brainstem.

Neurons within this area of the brain efferent output to the respiratory control muscles. Multiple afferent inputs induce modulation of the central respiratory center efferent outputs to the respiratory and airway muscles and lungs.

Among these inputs are signals from central and peripheral chemoreceptors, pulmonary stretch receptors, and cortical and reticuloactivating system neurons. Theophylline and caffeine have been shown to increase the central chemoreceptor ventilatory response to $\mathrm{CO}_{2}$ and decrease the number of apneic spells in premature babies.

\section{Physiologic Response to Respiratory Diseases}

Tachypnea (RR > 60/min): Rapid and shallow respirations are characteristic of parenchymal pathology, in which the elastic work of breathing is increased disproportionately to the resistive work of breathing.

Retractions: Subcostal, intercostal, and suprasternal are most striking, with increased negative intrathoracic pressure during inspiration. This occurs in extrathoracic airway obstruction as well as diseases of decreased compliance.

Inspiratory stridor is a hallmark of extrathoracic airwayobstruction.

Expiratory wheezing is characteristic of intrathoracic airway obstruction, either extrapulmonary or intrapulmonary. 
Grunting is produced by expiration against a partially closed glottis and is an attempt to maintain positive airway pressure during expiration for as long as possible. Such prolongation of positive pressure is most beneficial in alveolar diseases that produce widespread loss of FRC, such as in pulmonary edema, RDS and pneumonia.

\section{Respiratory Support in the Neonate}

The aim of respiratory support in the neonate is to maintain adequate gas exchange, minimize risk of lung injury, minimize haemodynamic impairment, avoid injury to other organs, and to reduce work of breathing.

\section{Oxygen and issues regarding use}

Oxygen as a drug provided in the neonate can be used to improve arterial oxygenation, cause pulmonary vasodilation, and to enhance systemic oxygen delivery.

Oxygen therapy works by increasing the fractional inspired concentration of oxygen $\left(\mathrm{FiO}_{2}\right)$ and the oxygen flow rate. The $\mathrm{FiO}_{2}$ determined by the concentration of supplemental oxygen, the flow rate of oxygen, oxygen delivery device, and the patient respiratory effort.

The concentration of oxygen varies depending on the source as follows: Room air $21 \%$, oxygen tank $100 \%$, and oxygen concentrator $>90 \%$ (varies with the oxygen concentrator indicator).

Oxygen delivery device that can be used include nasal cannula, nasal catheter, face mask, oxygen hood, oxygen tent, endotracheal/nasotracheal tube/tracheostomy

Nasal cannula: This is a low flow device that can deliver distending pressure. It is the least expensive and the $\mathrm{FiO}_{2}$ delivered is $24-40 \%$.

Nasal catheter: It is inserted into the oropharynx, and delivers a FiO2 similar to nasal cannula It could easily be clogged by secretions.

Face mask: There are various type which include the simple, partial rebreathing, non-rebreathing and the Venturi face mask.

Simple face mask: Allows unregulated flow of room air, and the $\mathrm{FiO}_{2}$ delivered is $35-50 \%$. However an increase flow rate is needed to prevent rebreathing.

Partial rebreather mask: Is a simple mask with an attached reservoir. Oxygen flow rate of 6-10L required to deliver $40-70 \% \mathrm{FiO}_{2}$ and a high flow is needed to prevent $\mathrm{CO}_{2}$ build up.

Non-rebreather face mask: Delivers highest $\mathrm{FiO}_{2}$ at $70-100 \%$

Venturi mask: This works based on the Venturi principle. It is the best method for delivering a specific and consistent $\mathrm{FiO}_{2}$. The mask can deliver $\mathrm{FiO}_{2}$ of $24-55 \%$ at flow rate of $4-10 \mathrm{~L}$. The masks are usually colour coded at $24 \%, 28 \%, 31 \%, 35 \%$, $40 \%$ and $50 \%$

Oxygen hood/ tent: has the added advantage of easy visualization.

\section{Monitoring Oxygen Therapy}

Clinical indices used include:

$$
\begin{aligned}
& \text { Oxygen saturation }\left(\mathrm{spO}_{2}\right) \\
& \text { Transcutaneous } \mathrm{PO} 2\left(\mathrm{tcPO}_{2}\right) \\
& \text { Transcutaneous } \mathrm{CO}\left(\mathrm{tcPCO}_{2}\right) \\
& \text { Arterial blood gases } \\
& \text { Central mixed venous } \mathrm{PO}_{2} \\
& \text { End tidal } \mathrm{CO}_{2}
\end{aligned}
$$

Complications of Oxygen Therapy: These include retinopathy of prematurity, bronchopulmonary dysplasia, absorption atelectasis, respiratory acidosis, ventilatory depression, suppression of erythropoeisis. Others are the complications related to the delivery device.

\section{Continuous Positive Airway Pressure}

The application of end-expiratory pressure is intended to prevent alveoli and/or terminal airways from collapsing to airlessness. Continuous positive airway pressure (CPAP) may be applied during spontaneous breathing or as positive end-expiratory pressure (PEEP) during mechanical ventilation. This usually requires pressures between 4 to $6 \mathrm{~cm} \mathrm{H}_{2} \mathrm{O}$ for $\mathrm{CPAP}$ and 3 to $8 \mathrm{~cm} \mathrm{H}_{2} \mathrm{O}$ for PEEP. The physiologic effects of CPAP/PEEP may vary depending on the underlying pulmonary pathology, although the primary goal is to prevent alveolar collapse.

In the surfactant-deficient state, alveoli will collapse at end-expiration unless a minimum distending pressure is maintained. CPAP of 3 to $4 \mathrm{~cm} \mathrm{H}_{2} \mathrm{O}$ will prevent alveolar collapse but will not recruit atelectatic alveoli. Opening pressures of 12 to $15 \mathrm{~cm}$ $\mathrm{H}_{2} \mathrm{O}$ are required to inflate collapsed alveoli. The infant will need to create a large distending airway pressure in the absence of CPAP. The shear forces from opening and closing of small airways may contribute to alveolar epithelial damage. CPAP theoretically could stimulate surfactant secretion. Also, maintenance of alveolar volume will reduce right-to-left shunting of blood through atelectatic alveoli, hence reducing oxygen needs. 
Indications: Its initial use was directed at RDS. However, can be used to reduce the need for ventilatory care in extreme preterms, and is used for the INSURE technique. It is also useful in recurrent apnoea of prematurity, and when weaning off conventional ventilation.

Benefits: It effectively maintains Functional Residual capacity, helps reduce infant work of breathing, reduces the need for intubation and mechanical ventilation, and reduces the incidence of chronic lung disease. It results in improved nonpulmonary outcomes (increase mean weight gain, mean length and head circumference at 36weeks post menstrual age).

Complications: This includes overinflation leading to increased work of breathing, air leak syndromes, carbon dioxide retention, decreased cardiac output with high values, complications from delivery device, and gastric distension.

\section{Mechanical Ventilation}

This is an invasive life support procedure. The goal is to optimise both gas exchange and clinical status at minimum $\mathrm{FiO}_{2}$ and ventilatory pressures/ tidal volumes.

\section{Conventional Ventilation}

Ventilatory modes: Untriggered or triggered. Untriggered Mode: Consists of intermittent mandatory ventilation (IMV) and intermittent positive pressure ventilation (IPPV).

Intermittent Mandatory Ventilation: Provides fixed rate of mechanical ventilation and allows spontaneous breathing between mechanical breathes from continuous flow of oxygen.

Triggered ventilation: Consists of flow trigger and pressure trigger

Triggered modes: Types are the assist/control mode, synchronized intermittent mandatory ventilation, pressure support ventilation, flow sensitive ventilation, and volume timed ventilation.

Assist/Control (A/C): This modality involves either the delivery of a synchronized mechanical breath each time a spontaneous patient breath is detected (ASSIST), or in the event that the patient fails to exhibit spontaneous resp. effort, the ventilator delivers a mechanical breath at a regular rate (CONTROL).
Synchronized Intermittent Mandatory Ventilation (SIMV): Is a ventilatory mode in which the mechanically delivered breaths are synchronized to the onset of spontaneous patient breaths, but at a lower rate. The patient may breathe spontaneously between mechanical breaths from the continuous flow in the ventilatory circuit.

Pressure Support Ventilation (PSV): It is a mode of ventilation that has no set rate and only supports the patient's own spontaneous effort. It is primarily a weaning mode. The patient controls RR, Ti and peak insp. Flow, while the ventilator controls only PIP. This system synchronizes inspiration by sensing patient effort, and also synchronizes expiration by terminating inspiration in response to a decline in airway flow. This results in complete synchronization of the functioning of the baby and the ventilator throughout the entire respiratory cycle.

Flow Sensitive Ventilation (FSV): Inspiration is triggered by changes in flow and ends not according to time but according to airway flow changes. During inspiration, the ventilator records the peak expiratory flow rate and subsequently terminates inspiration when the inspiratory flow decreases to $5-10 \%$ of peak flow. This enables both inspiratory and expiratory synchrony. Benefits of FSV include total breath synchronization, decreased work of breathing, less sedation, more efficient tidal volume delivery, improved gas exchange, and fewer complications.

\section{Newer modalities of Mechanical Ventilation}

Volume guaranteed (VG)

Volume associated pressure support (VAPS)

Pressure regulated volume control (PRVC)

Proportional assist ventilation (PAV)

\section{Volume Guaranteed ( $V G$ )}

This is a pressure-limited, time or flowcycled, volume-targeted form of ventilation. The microprocessor compares exhaled tidal volume of the previous breath to the desired target and adjusts the working pressure up or down to try to achieve the target tidal volume. There is limit of pressure increment from one breath to the next to a maximum of $3 \mathrm{~cm} \mathrm{H} \mathrm{H}_{2} \mathrm{O}$ to avoid overcorrection. Thus, several breaths may be needed to reach the target tidal volume after a sudden change. The VG mode cannot increase pressure higher than set pressure limit.

\section{Benefits of VG}

Maintenance of constant tidal volumes in the face of changing compliance, resistance and changing ET- tube leak

Prevention of overdistention and volutrauma 
Automatic lowering of pressure support level during weaning (auto-weaning). As the patient's lungs improve and compliance increases, peak inspiratory pressure is weaned automatically.

Indications for VG: Virtually any infant requiring mechanical ventilation especially when lung mechanics are likely to change, or in patients with heterogenous lung disease because of differing time constant throughout lung parenchyma

\section{High Frequency Ventilation}

HFV is a form of mechanical ventilation that uses small tidal volumes, sometimes less than anatomic dead space, and extremely rapid ventilator rates.

\section{HFV in comparison to conventional IMV}

HFV delivers at high frequency: 300-1200/min=5$20 \mathrm{HzU}$ and utilizes very small tidal volumes and can detect incomplete inspiration and expiration. Causes dampening of the oscillations along the airways and ensures a nearly constant alveolar pressure. Also, HFV has the ability to independently manage ventilation and oxygenation, while ensuring the safe use of mean airway pressure that is higher than that generally used during conventional mechanical ventilation.

Types of HFV

HFFI (Higher-Frequency Flow interrupting Ventilation)

HFJV (High- Frequency Jet Ventilation)

HFOV (High-Frequency Oscillatory Ventilation)

Indications for HFOV: Rescue therapy and air leak syndromes.

\section{Clinical Applications of Mechanical Ventilation}

CPAP: Mildly affected neonates with RDS: start at 4-6 $\mathrm{cm} \mathrm{H}_{2} \mathrm{O}$ and increase gradually to a maximum of 7-8 $\mathrm{cm} \mathrm{H}_{2} \mathrm{O}$. It is titrated by: clinical assessment of retractions, respiratory rates, and oxygen saturation.

\section{Neonatal Pulmonary Physiology By Disease State.}

Strategies need to be developed for the various disease states requiring ventilation in the neonate. Setting parameters are required for the inspiratory flow rate, peak inspiratory pressure, positive end expiratory pressure, oxygen concentration, inspiratory time, expiratory time, trigger volume. Derived parameters are the $\mathrm{FiO} 2$, mean airway pressure, flow parameters, tidal volume, and the respiratory rate.
1. Ventilatory strategy in RDS Ensure an inspiratory flow rate $7-12 \mathrm{~L} / \mathrm{min}$, peak inspiratory pressure $20-25 \mathrm{cmH}_{2} \mathrm{O}$ and positive end expiratory pressure $4-5 \mathrm{cmH}_{2} \mathrm{O}$. The inspiratory time should be $0.5 \mathrm{~s}$ while the expiratory time is set at $1.0 \mathrm{~s}$. Trigger volume mode preferred. Alternative strategy is with high frequency ventilation.

2. Ventilatory strategy in MAS

There is a high risk of pneumothorax because of ball valve effect, thus a low PEEP should be utilized to splint the airways. If airway resistance is high, a slow rate, moderate pressure strategy should be utilized. If pneumonitis is more prominent, more rapid rates can be utilized. HFV can be used with failed conventional ventilation or air leaks.

3. Ventilatory strategy in air leaks

Goal is to reduce the mean airway pressure (MAP) to as low as possible and rely on $\mathrm{FiO}_{2}$ to improve oxygenation. HFOV is the modality of choice. Maintain MAP, do not use sigh maneuver. Use low PEEP

4. Ventilatory strategy in apnoea with normal lungs Ensure low gas flow, low PIP $10-18 \mathrm{cmH}_{2} \mathrm{O}$, low PEEP 3-4 $\mathrm{cmH}_{2} \mathrm{O}$, and normal rates 30 40breaths/min

Oxygenation Index: This is an index of disease severity

$$
\mathrm{OI}=\frac{\mathrm{PAW} \times \mathrm{FiO} 2}{\mathrm{PaO} 2}
$$

An index greater than 15 indicates severe respiratory compromise, while an index greater than 40 on multiple occasions indicate mortality $>80 \%$

\section{Complications of Mechanical Ventilation}

This includes ventilation induced lung injury, ventilation induced pneumonia, air leak syndromes, traumatic injury to large airways and endotracheal tube complications.

\section{Ventilator Induced Lung Injury (VILI)}

There are four mechanisms of VILI: Barotrauma (high airway pressure), volutrauma (large tidal volume), atelectotrauma (alveolar collapse and reexpansion), and biotrauma (increased inflammation).

Volutrauma caused by mechanical overdistension leads to alveolar epithelial cell damage, alveolar protein damage, altered lymphatic flow, hyaline membrane formation, and inflammatory cell influx in the lungs. The precise tidal volume required to minimize volutrauma is not known. Therefore efforts to limit tidal volume may be a beneficial practice in the neonatal intensive care unit. 
Ventilator Associated Pneumonia (VAP)

This is defined as pneumonia in mechanically ventilated patients that develops $\geq 48 \mathrm{~h}$ after the patient has been placed on mechanical ventilation. VAP is the second most common hospital acquired infection among neonatal intensive care unit (NICU) patients. This is often caused by organisms such as Pseudomonas aeruginosa (the most common), Staphylococcus aureus, Enterobacter species, and Klebsiella pneumoniae

\section{Diagnostic criteria for VAP}

Worsening gas exchange (oxygen desaturation or increased oxygen or ventilatory requirements) and 3 of the following:

Temperature instability with no obvious cause. Leukopenia or leukocytosis with left shift. Increased pulmonary secretions or greater need for suctioning.

Examination reveals apnea, tachypnea, nasal flaring with chest retractions, grunting. Wheezing, rales, rhonchi may be present, with either bradycardia or tachycardia.

Strategies to reduce VAP will include to prevent contamination of equipment, ensure endotracheal tube care, and to minimize duration of intubation.

\section{Air-leaksyndromes}

These include: Pneumothorax, pneumomediastinum, pneumopericardium, pulmonary interstitial emphysema, and subcutaneous emphysema.

Risk factors for air-leak syndromes in neonates are extreme low birth weight, endotracheal tube displacement, using long inspiratory time $(>0.5$ $\mathrm{sec}), \uparrow \mathrm{PIP}, \uparrow \mathrm{Vt}$. Also, increase in clinical interventions including suction procedures, chest radiography, reintubation, bag \& mask ventilation and chest compressions are risk factors. Other risk factors are MAS, RDS, pulmonary hypoplasia.

Tracheal injury and endotracheal tube related complications

These include subglottic stenosis, tracheal perforation, palatal deformities, vocal cord avulsion, laryngeal oedema, subglottic cysts, necrotizing tracheitis and septal injury.

Chronic lung disease is defined as the need for supplemental oxygen beyond 28days of postnatal age or oxygen dependency at 36 weeks postmenstrual age.
Retinopathy of prematurity is a vaso-proliferative retinal disorder that decreases with gestational age. Approximately $65 \%$ of infants with a birth weight less than $1250 \mathrm{~g}$ and $80 \%$ of those with a birth weight less than $1000 \mathrm{~g}$ will develop some degree of retinopathy of prematurity.

\section{Extracorporeal Membrane Oxygenation}

This can be defined as a mechanical means of providing oxygen delivery and carbon dioxide removal for patients who have cardiac and/or respiratory failure. May be veno-arterial or venovenous.

\section{Indication}

Critically ill term and late preterm newborns with reversible respiratory and/or cardiac failure who have failed appropriate maximal medical management.

In conditions such as meconium aspiration syndrome, respiratory distress syndrome, persistent pulmonary hypertension of the newborn, pneumonia, sepsis, severe rhythm disturbances, neonatal cardi0myopathies

Specific indications

Respiratory criteria: OI $>30-40$ for 4 hours, acute deterioration with intractable hypoxaemia, barotrauma with severe air leak not responsive to HFOV

Cardiovascular/oxygen delivery criteria:Cardiac arrest, plasma lactate $>45 \mathrm{mg} / \mathrm{dl}$ with metabolic acidosis not improving or escalating with despite adequate medical care, mixed venous saturation $<55 \%$ for 1 hour

Contraindications

These include: Gestational age less than 32 weeks and/ or birth weight less than $1800 \mathrm{~g}$, mechanical ventilation beyond 10-14 days due to likely irreversible lung disease, intraventricular hemorrhage greater than grade I, and coagulopathy unlikely to resolve with transfusion. Others are severe congenital anomalies, uncorrectable cardiac lesions, $\mathrm{CDH}$ with $\mathrm{OI}>45$, hypoxic ischaemic encephalopathy, and plasma lactate $>225 \mathrm{mg} / \mathrm{dl}$.

\section{Complications}

These are acute renal failure, hypertension, haemolysis, seizures, hypotension, CNS infarction, intracranial haemorrhages, sepsis, surgical bleeding, pulmonary haemorrhage, disseminated intravascular coagulopathy (DIC), and brain death. Others are cannula problems, air embolism, pump failure, and clot formation.

Supportive Care: This involves the use of surfactant therapy, inhaled nitric oxide, heliox, ensuring 
adequate tissue perfusion by optimizing cardiovascular function and total parenteral nutrition.

\section{To be more practical where do we stand?}

There is the need to establish mechanical ventilatory care in our centers. Requirements are:

Manpower: Nursing staff, doctors, laboratory staff, radiographers, need for training.

Materials: Functioning ventilators, regular oxygen supply, regular power supply, regular water supply, a mobile x-ray machine, pulse oximeters, complete patient monitors (BP, HR, Temp, $\mathrm{O}_{2}$ saturation),
Arterial blood gas analyzers, flow sensors, oxygen sensors, surfactant, total parenteral nutrition (TPN), and disposable supplies (endotracheal tubes, catheters, chest tubes, etc).

\section{Acknowledgement}

Reproduced with kind permission of the department of Paediatrics and Child Health of the University of Ilorin Teaching Hospital, Ilorin Nigeria owners of the Ilorin Paediatric Digest 2010.

\section{References}

1. Gomella LT. Neonatology: Management, Procedures, On-call Problems, Diseases, and Drugs. $6^{\text {th }}$ ed. New York:

McGraw Hill, 2009.

2. Cloherty JP, Eichenwald EC, Stark AR. Manual of neonatal care. $6^{\text {th }}$ ed. Philadelphia: Lippincott Williams, 2008.

3. Fox G, Hoque N, Watts T. Oxford Handbook of Neonatology. $1^{\text {st }}$ ed. Oxford: Oxford University Press, 2010.

4. Helfaer MA, Nichols DG. Rogers' Handbook of Pediatric Intensive Care. $4^{\text {th }}$ ed. Philadelphia: Lippincott Williams \& Wilkins, 2009.
5. Chatburn RL. Fundamentals of Mechanical Ventilation. $1^{\text {st }}$ ed. Ohio: Mandu Press, 2003.

6. Tobin MJ. Principles and Practice of Mechanical Ventilation. $1^{\text {st }}$ ed. New York: McGraw Hill, 1994.

7. El Sallab S. Modes of conventional mechanical ventilation. Text of a lecture delivered at the Advanced Neonatology Workshop in Mansoura, Egypt, November 2010.

8. Khashaba M. Pulmonary Physiology. Text of a lecture delivered at the Advanced Neonatology Workshop in Mansoura, Egypt, November 2010.
9. Shouman B. Complications of mechanical ventilation. Text of a lecture delivered at the Advanced Neonatology Workshop in Mansoura, Egypt, November 2010.

10. Ganong WF, editor. Review of Medical Physiology. 21st ed. San Francisco: Lange Medical Books/McGrawHill Medical Publishing Division 2003.

11. Sadler TW. Langman's Medical Embryology, $9^{\text {th }}$ edition, 2004; 275284. 